\title{
ON THE ASYMPTOTIC BEHAVIOUR OF ALGEBROID FUNCTIONS OF EXTREMAL GROWTH
}

\author{
By TSUneo SATo
}

1. Valiron [5] and Wahlund [7] established that an entire function $g(z)$ of order $\lambda$ satisfies

$$
\varlimsup_{r \rightarrow \infty} \frac{N(r, 0)}{\log M(r, g)} \geqq \frac{\sin \pi \lambda}{\pi \lambda} \quad(0 \leqq \lambda<1)
$$

This classical result was extended by Ozawa [3] to $n$-valued entire algebroid functions. In a recent paper of Williamson [8] it has been sharpened to the following:

Let $g$ be an entire function of lower order $\mu<1$.

Then

$$
\varlimsup_{r \rightarrow \infty} \frac{N(r, 0)}{\log M(r, g)} \geqq \frac{\sin \pi \mu}{\pi \mu}(0 \leqq \mu<1) .
$$

And Williamson has also obtained a complete answer to the question, what can be said about the asymptotic behaviour of an entire function for which equality holds in (1.1).

The purpose of this paper is to extend Williamson's theorem to $n$-valued entire algebroid functions of lower order $\mu<1$. Let $f(z)$ be an $n$-valued entire algebroid function, $f_{\nu}(z)$ the $\nu$-th determination of $f(z), N(r ; a, f)$ the countingfunction of $f(z)$ and $M(r, f)$ the maximum modulus of $f$ on $|z|=r$ such that

$$
\log M(r, f)=\max _{|z|=r} \max _{1 \leqq \nu \leqq n} \log \left|f_{\nu}(z)\right| .
$$

We shall prove the following extension of Williamson's theorem :

THEOREM 1. Let $f(z)$ be an n-valued transcendental entire algebroid function of lower order $\mu<1$. Then there is at least one $a_{\nu}$ among $n$ different finte numbers $a_{\jmath}, j=1, \cdots, n$, satısfying

$$
\varlimsup_{r \rightarrow \infty} \frac{n N\left(r ; a_{\nu}, f\right)}{\log M(r, f)} \geqq \frac{\sin \pi \mu}{\pi \mu} .
$$

Next we shall get the following theorem that gives a precise meaning to the asymptotic behaviour of an $n$-valued entire algebroid function for which equality holds in (1.2).

Receıved October 31, 1980. 
THEOREM 2. Let $f(z)$ be an n-valued transcendental entire algebroid function of lower order $\mu<1$ and assume that the equality

$$
\varlimsup_{r \rightarrow \infty} \frac{n N\left(r ; a_{\nu}, f\right)}{\log M(r, f)}=\frac{\sin \pi \mu}{\pi \mu}
$$

holds for all $n$ different finte numbers $a_{\nu}, \nu=1, \cdots, n$. Let $\left\{r_{m}\right\}$ be a sequence of Pólya peaks of order $\mu$ of $\log M(r, f)$. Then there exist three positive sequences $\left\{R_{m}^{\prime}\right\},\left\{R_{m}^{\prime \prime}\right\},\left\{\tilde{\varepsilon}_{m}\right\}$ such that

$$
\lim _{m \rightarrow \infty} R_{m}^{\prime}=\lim _{m \rightarrow \infty} \frac{R_{m}^{\prime \prime}}{r_{m}}=\infty ; \quad \lim _{m \rightarrow \infty} \frac{R_{m}^{\prime \prime}}{r_{m}}=\lim _{m \rightarrow \infty} \tilde{\varepsilon}_{m}=0,
$$

and such that the inequalities

$$
R_{m}^{\prime} \leqq t \leqq R_{m}^{\prime \prime} \quad\left(m>m_{0}\right),
$$

imply the inequalities

and

$$
\begin{gathered}
\left(\frac{t}{r_{m}}\right)^{\mu}\left(1+\tilde{\varepsilon}_{m}\right)^{-1} \leqq \frac{\log M(t, f)}{\log M\left(r_{m}, f\right)} \leqq\left(\frac{t}{r_{m}}\right)^{\mu}\left(1+\tilde{\varepsilon}_{m}\right), \\
\frac{\sin \pi \mu}{\pi \mu}-\tilde{\varepsilon}_{m} \leqq \frac{n N\left(t ; a_{\nu}, f\right)}{\log M(t, f)} \leqq \frac{\sin \pi \mu}{\pi \mu}+\tilde{\varepsilon}_{m}
\end{gathered}
$$

$$
\frac{\sin \pi \mu}{\pi \mu}-\tilde{\varepsilon}_{m} \leqq \frac{n n\left(t ; a_{\nu}, f\right)}{\log M(t, f)} \leqq \frac{\sin \pi \mu}{\pi \mu}+\tilde{\varepsilon}_{m}
$$

for some $a_{\nu}, \nu=1, \cdots, n$.

2. Preliminaries. Let $f(z)$ be an $n$-valued transcendental algebroid function defined by an irreducible equation

$$
f^{n}+A_{1}(z) f^{n-1}+\cdots+A_{n-1}(z) f+A_{n}(z)=0,
$$

where $A_{1}, \cdots, A_{n}$ are entire functions without common zeros. Let $f_{2}(z)$ be the $\nu$-th determination of $f(z)$.

We put

$$
\begin{aligned}
& A(z)=\max \left(1,\left|A_{1}\right|, \cdots,\left|A_{n}\right|\right. \\
& g(z)=\max \left(1,\left|g_{1}\right|, \cdots,\left|g_{n}\right|\right) \\
& g_{\nu}(z)=F\left(z, a_{\nu}\right), \quad \nu=1, \cdots, n
\end{aligned}
$$

where $F(z, f)=0$ is the defining equation of $f$.

Then we have

$$
\begin{aligned}
\log M(r, f) & =\max _{|z|=r} \max _{1 \leqq \nu \leqq n} \log \left|f_{\nu}(z)\right| \\
& \leqq \max _{|z|=r} \max _{1 \leqq \nu \leqq n} \log ^{+}\left|f_{\nu}(z)\right|
\end{aligned}
$$




$$
\leqq \max _{|z|=r} \sum_{1}^{n} \log ^{+}\left|f_{\nu}(z)\right|
$$

and by Valiron's argument [6]

$$
\sum_{1}^{n} \log ^{+}\left|f_{:}(z)\right| \leqq \log A(z)+O(1) \leqq \log g(z)+O(1) .
$$

Further we have

$$
\begin{aligned}
\max _{|z|=r} \log g(z) & =\log \max _{|z|=r} g(z)=\log \max _{1 \leqq \nu \geqq n} \max _{|z|=r}\left|g_{\nu}(z)\right| \\
& =\max _{1 \leqq \nu \leqq n} \log M\left(r, g_{\nu}\right) .
\end{aligned}
$$

Hence we get

$$
\log M(r, f) \leqq \max _{1 \leqq \nu \leqq n} \log M\left(r, g_{\nu}\right)+O(1)
$$

And we remark

$$
\log M\left(r, g_{\nu}\right) \leqq \log M(r, f)+O(1)
$$

3. Proof of Theorem 1. We shall give a proof of Theorem 1 according to Edrei's idea [1], using his well-known representation. Let $g_{\nu}$ be $F\left(z, a_{\nu}\right)$. Denote its zero by $\left\{b_{k}\right\}$. Then we can write

$$
\log \left|g_{\nu}\left(r e^{\imath \theta}\right)\right| \leqq \sum_{0<\left|b_{k}\right| \leqq R} \log \left|1-\frac{r e^{\imath \theta}}{b_{k}}\right|+A \frac{r}{R} \log M\left(2 R, g_{\nu}\right),
$$

provided that $|z|=r \leqq R / 2$. Then we have

$$
\log M\left(r, g_{\nu}\right) \leqq r \int_{0}^{R} N\left(t ; 0, g_{\nu}\right) \frac{d t}{(t+r)^{2}}+A \frac{r}{R} \log M\left(2 R, g_{\nu}\right)+O(1) .
$$

Hence we obtain from (2.1) that

$$
\begin{aligned}
\log M(r, f) & \leqq r \max _{1 \leqq \nu \leqq n} \int_{0}^{R} N\left(t ; 0, g_{\nu}\right) \frac{d t}{(t+r)^{2}}+A \frac{r}{R} \log M(2 R, f) \\
& =r \max _{1 \leqq \nu \leqq n} n \int_{0}^{R} N\left(t ; a_{\nu}, f\right) \frac{d t}{(t+r)^{2}}+A \frac{r}{R} \log M(2 R, f) .
\end{aligned}
$$

Assume that for all $\nu$

$$
\varlimsup_{r \rightarrow \infty} \frac{n N\left(r ; a_{\nu}, f\right)}{\log M(r, f)}<\frac{\sin \pi \mu}{\pi \mu}
$$

Then

for $r \geqq r_{0}$. Thus

$$
\frac{n N\left(r ; a_{\nu}, f\right)}{\log M(r, f)}<\frac{\sin \pi \mu}{\pi \mu}-\varepsilon=U, \quad \varepsilon>0
$$

$$
\log M(r, f)<r U \int_{r_{0}}^{R} \log M(t, f) \frac{d t}{(t+r)^{2}}+A \frac{r}{R} \log M(2 R, f)+O(1) .
$$


Now we make use of the notion of Pólya peaks of order $\mu$ of $\log M(t, f)$. It is possible to find three positive sequences $\left\{r_{m}^{\prime}\right\},\left\{r_{m}^{\prime \prime}\right\},\left\{\varepsilon_{m}\right\}$ such that

$$
\lim _{m \rightarrow \infty} r_{m}^{\prime}=\lim _{m \rightarrow \infty} \frac{r_{m}^{\prime \prime}}{r_{m}}=\infty, \quad \lim _{m \rightarrow \infty} \frac{r_{m}^{\prime}}{r_{m}}=\lim _{m \rightarrow \infty} \varepsilon_{m}=0
$$

and such that the inequalities

imply that

$$
r_{m}^{\prime} \leqq t \leqq r_{m}^{\prime \prime} \quad\left(m>m_{0}\right)
$$

$$
\log M(t, f) \leqq\left(1+\varepsilon_{m}\right)\left(\frac{t}{r_{m}}\right)^{\mu} \log M\left(r_{m}, f\right) .
$$

We deduce from (3.2) on setting

$$
r=r_{m}, \quad R=\frac{r_{m}^{\prime \prime}}{2}
$$

that

$$
\begin{aligned}
\log M\left(r_{m}, f\right)< & r_{m} U \int_{r_{m}^{\prime}}^{r_{m}^{\prime}} \log M(t, f) \frac{d t}{\left(t+r_{m}\right)^{2}} \\
& +r_{m} U \int_{r_{0}}^{r_{m}^{\prime}} \log M(t, f) \frac{d t}{\left(t+r_{m}\right)^{2}}+A \frac{r_{m}}{r_{m}^{\prime \prime}} \log M\left(r_{m}^{\prime \prime}, f\right)+O(1) .
\end{aligned}
$$

By (3.3) the second integral and third term are dominated respectively by

$$
\begin{aligned}
& U \log M\left(r_{m}^{\prime}, f\right) \int_{r_{0} / r_{m}}^{r_{m}^{\prime} / r_{m}} \frac{d x}{(x+1)^{2}} \leqq U-\frac{r_{m}^{\prime}}{r_{m}} \log M\left(r_{m}^{\prime}, f\right)=o\left(\log M\left(r_{m}, f\right)\right) \quad(m \rightarrow \infty), \\
& \frac{r_{m}}{r_{m}^{\prime \prime}} \log M\left(r_{m}^{\prime \prime}, f\right) \leqq\left(1+\varepsilon_{m}\right) \frac{r_{m}}{r_{m}^{\prime \prime}}\left(\frac{r_{m}^{\prime \prime}}{r_{m}}\right)^{\mu} \log M\left(r_{m}, f\right)=o\left(\log M\left(r_{m}, f\right)\right) \quad(m \rightarrow \infty)
\end{aligned}
$$

since $\mu<1$. Thus in veiw of (3.4) we have

$$
\begin{aligned}
\log M\left(r_{m}, f\right)< & r_{m} U\left(1+\varepsilon_{m}\right) \log M\left(r_{m}, f\right) \int_{r_{m}^{\prime}}^{r_{m}^{\prime \prime}}\left(\frac{t}{r_{m}}\right)^{\mu} \frac{d t}{\left(t+r_{m}\right)^{2}} \\
& +o\left(\log M\left(r_{m}, f\right)\right) .
\end{aligned}
$$

Dividing this by $\log M\left(r_{m}, f\right)$, setting $x=t / r_{m}$ in the integral, and letting $m \rightarrow \infty$, we obtain

$$
1 \leqq U \int_{0}^{\infty} x^{\mu} \frac{d x}{(x+1)^{2}}=U \frac{\pi \mu}{\sin \pi \mu}
$$

and by definition of $U$ we have

$$
\begin{aligned}
& 1 \leqq U \frac{\pi \mu}{\sin \pi \mu}=\left(\frac{\sin \pi \mu}{\pi \mu}-\varepsilon\right) \frac{\pi \mu}{\sin \pi \mu} \\
& \quad=1-\varepsilon \frac{\pi \mu}{\sin \pi \mu}<1,
\end{aligned}
$$

which is a contradiction. Hence Theorem 1 follows. 
4. Example. Now we can consider equality parts in the inequality (2.1). Let $f(z ; \lambda)$ be the Lindelöf function

$$
f(z ; \lambda)=\prod_{\nu=1}^{\infty}\left(1+\frac{z}{b_{\nu}}\right), \quad b_{\nu}=\nu^{1 / \lambda}, \quad \nu=1,2,3, \cdots .
$$

Let $h_{\alpha}(z)=f\left(\alpha^{1 / \lambda}(z+c) ; \mu\right)$. The asymptotic behaviour of $f(z ; \mu)$ is completely known (cf. $[4$, p. 18]). In particular, it is easy to verify that $f(z ; \mu)$ has order $\lambda$, lower order $\mu=\lambda$, and further that

$$
\log h_{\alpha}(z)=\frac{\pi \alpha}{\sin \pi \mu} z^{\mu}(1+\varepsilon(z)),
$$

where $\varepsilon(z) \rightarrow 0$, uniformly as $z \rightarrow \infty$ in the angle

and hence

$$
|\arg z| \leqq \pi-\eta \quad(0<\eta<\pi),
$$

$$
\log M\left(r, h_{\alpha}\right) \sim \frac{\pi \alpha}{\sin \pi \mu} r^{\mu} \quad(r \rightarrow \infty) .
$$

Let $n(r, 0)$ denote the counting-function associated with $h_{\alpha}(z)$; clearly

and hence

$$
n(r, 0) \sim \alpha r^{\mu} \quad(r \rightarrow \infty),
$$

$$
N(r, 0) \sim \frac{\alpha r^{\mu}}{\mu} \quad(r \rightarrow \infty)
$$

Now we consider

$$
F(z, f) \equiv f^{n}-h_{\alpha}(z) f^{n-1}+h_{\alpha}(z)-e^{\imath \theta}=0 \quad(\theta \text { is irrational. })
$$

which is irreducible.

The irreducibility of this equation (4.2) can be showed the following:

Suppose that

then

$$
F(z, f) \equiv A(z, f) B(z, f),
$$

$$
F\left(z, a_{\nu}\right)=A\left(z, a_{\nu}\right) B\left(z, a_{\nu}\right)=\text { const. }
$$

Hence we can have two cases.

Or

Case 1) $A\left(z, a_{\nu}\right), B\left(z, a_{\nu}\right)$ are constants together.

Case 2) $A\left(z, a_{\nu}\right)=c_{1} e^{H}, \quad B\left(z, a_{\nu}\right)=c_{2} e^{-H}$,

where $H$ is an entire function. For if $A\left(z, a_{\nu}\right)=0$ has zeros, then $B\left(z, a_{\nu}\right)=0$ must have poles, which contradicts to that $B\left(z, a_{\nu}\right)$ is an entire algebroid function.

Suppose that $p \leqq q$, then $A(z, f), B(z, f)$ have exceptional values with respective numbers $2 p-1,2 q-1$ with exceptional values of first kind and second kind combined.

Hence, assume that $2 p-1<n-1$, then a contradiction follows, we have 
thus

$$
2 p-1 \geqq n-1
$$

$$
2 p \geqq n \text {. }
$$

On the other hand $p \leqq q$ and $p+q=n$. This leads us to the following fact:

$$
p=q=\frac{n}{2} .
$$

This implies

$$
2 p-1=n-1 .
$$

Thus exceptional values of first kind number $2 p-1, A(z, f)=0$ has the number $p-1$ of exceptional values of first kind. Consequently $f$ has a positive integral order in view of the estimation of $K(f)$ by Toda.

Similarly we can deduce that the order of $f$ is a positive integer from $B(z, f)=0$.

By the positive integrity of order of $f$ we have a contradiction. This contradiction gives that the equation (4.2) is irreducible.

Let $f_{\alpha}(z)$ be an entire algebroid function defined by (4.2). Then we have the lower order of $f_{\alpha}(z)$ is $\mu=\lambda$. Denoting by $f_{\nu}(z)$ the $\nu$-th determination of $f_{\alpha}(z)$ and noting that $-h_{\alpha}(z)=\Sigma f_{\nu}(z)$, we have $\left|h_{\alpha}(z)\right| \leqq n \max _{1 \leqq \nu \leqq n}\left|f_{\nu}(z)\right|$ and consequently

$$
\log ^{+} M\left(r, h_{\alpha}\right) \leqq \log ^{+} M\left(r, f_{\alpha}\right)+\log n .
$$

Now by choosing $a_{\nu}$ suitably, for example

$$
a_{\nu}=\exp \left(2 \pi \nu i / n+\theta_{\imath} / n\right), \quad \nu=1, \cdots, n .
$$

we can say that the defining equation $g_{\nu} \equiv F\left(z, a_{\nu}\right)$ gives

$$
N\left(r ; a_{\nu}, f_{\alpha}\right)=\frac{1}{n} N\left(r ; 0, g_{\nu}\right)=\frac{1}{n} N\left(r ; 0, h_{\alpha}\right) \sim \frac{\alpha r^{\mu}}{n \mu} \quad(r \rightarrow \infty)
$$

for all $\nu$. Therefore it follows from (4.1), (4.3) and (4.4) that

$$
\frac{\sin \pi \mu}{\pi \mu}=\varlimsup_{r \rightarrow \infty} \frac{N\left(r ; 0, h_{\alpha}\right)}{\log M\left(r, h_{\alpha}\right)} \geqq \varlimsup_{r \rightarrow \infty} \frac{n N\left(r ; a_{\nu}, f_{\alpha}\right)}{\log M\left(r, f_{\alpha}\right)} .
$$

On the other hand we can get

$$
\varlimsup_{r \rightarrow \infty} \frac{n N\left(r ; a_{\nu}, f_{\alpha}\right)}{\log M\left(r, f_{\alpha}\right)} \geqq \frac{\sin \pi \mu}{\pi \mu}
$$

in view of (4.4) combining with Theorem 1. Thus from (4.5) we obtain

$$
\varlimsup_{r \rightarrow \infty} \frac{n N\left(r ; a_{\nu}, f_{\alpha}\right)}{\log M\left(r, f_{\alpha}\right)}=\frac{\sin \pi \mu}{\pi \mu}, \quad \nu=1, \cdots, n .
$$

Therefore there exist $n$ different finite numbers $a_{\nu}, \nu=1, \cdots, n$, which always satisfy the result that equality holds in (1.2) respectively. 
5. The proof of Theorem 2. Edrei-Williamson's argument does work on our case. We shall sketch very briefly the steps of the proof. First, we show that if equality holds in (1.2) for all $a_{\nu}(\nu=1, \cdots, n)$, then, for every fixed $\sigma>0$,

$$
\varlimsup_{m \rightarrow \infty} \frac{\log M\left(\sigma r_{m}, f\right)}{\log M\left(r_{m}, f\right)}=\sigma^{\mu} .
$$

Note that (3.4) implies that

$$
\varlimsup_{m \rightarrow \infty} \frac{\log M\left(\sigma r_{m}, f\right)}{\log M\left(r_{m}, f\right)} \leqq \sigma^{\prime \prime} .
$$

Thus, if (5.1) were false, there would exist some $\sigma>0$, some $\delta(0<\delta<1)$, and some unbounded sequence $\Lambda$ of positive integers such that

$$
\frac{\log M\left(\sigma r_{m}, f\right)}{\log M\left(r_{m}, f\right)}<\sigma^{\mu} \delta^{2 \prime \prime} \quad(m \in \Lambda) .
$$

Now by (3.1), in place of (3.2),

$$
\log M(r, f) \leqq r\left(\chi+\xi_{m}\right) \int_{0}^{R} \log M(t, f) \frac{d t}{\left(t+r_{m}\right)^{2}}+A \frac{r}{R} \log M(2 R, f)+O(1)
$$

in view of

$$
\chi=\frac{\sin \pi \mu}{\pi \mu}=\varlimsup_{r \rightarrow \infty} \frac{n N\left(r ; a_{\nu}, f\right)}{\log M(r, f)} \quad(\nu=1,2, \cdots, n),
$$

where $\left\{\hat{\xi}_{m}\right\}$ is a suitable sequence tending to zero as $m \rightarrow \infty$.

Similar to the case of (3.5), we obtain

$$
\log M\left(r_{m}, f\right) \leqq r_{m}\left(\chi+\xi_{m}\right) \int_{r_{m}^{\prime}}^{r_{m}^{\prime \prime}} \log M(t, f) \frac{d t}{\left(t+r_{m}\right)^{2}}+o\left(\log M\left(r_{m}, f\right)\right) \quad(m \rightarrow \infty)
$$

on using of the hypothesis of Theorem 2 .

Let $I_{1}\left(r_{m}\right), I_{2}\left(r_{m}\right), I_{3}\left(r_{m}\right)$ respectively denote the portion of the integral in (5.4) over the intervals $\left[r_{m}^{\prime}, \sigma \delta r_{m}\right],\left[\sigma \delta r_{m}, \sigma r_{m}\right],\left[\sigma r_{m}, r_{m}^{\prime \prime}\right]$. Then, by the same manner of the discussion [8],

$$
I_{1}\left(r_{m}\right)+I_{3}\left(r_{m}\right) \leqq\left(1+\varepsilon_{m}\right) \log M\left(r_{m}, f\right)\left\{\int_{0}^{\sigma \delta}+\int_{\sigma}^{\infty}\right\} x^{\mu} \frac{d x}{(x+1)^{2}} .
$$

And (5.3) implies that, for $m \in \Lambda$,

$$
I_{2}\left(r_{m}\right)<\log M\left(r_{m}, f\right) \delta^{2 \mu} \sigma^{\mu} \int_{\sigma \delta}^{\sigma} \frac{d x}{(x+1)^{2}} .
$$

Thus, for $m \in \Lambda$, by combining the above two inequalities, (5.4) yields

where

$$
1+o(1) \leqq\left(1+\varepsilon_{m}\right)\left(\chi+\xi_{m}\right)\left\{\frac{\pi \mu}{\sin \pi \mu}-K\right\},
$$

$$
K=\sigma^{\mu} \delta^{\mu}\left(1-\delta^{\mu}\right) \int_{\sigma \delta}^{\sigma} \frac{d x}{(x+1)^{2}}>0 .
$$

Letting $m \rightarrow \infty(m \in \Lambda)$ here, we find that 


$$
1 \leqq \chi\left\{\frac{\pi \mu}{\sin \pi \mu}-K\right\},
$$

which is a contradiction. Thus, assumption (5.3) is false and (5.1) true.

It now follows from (5.1) and Edrei's lemma [2] that there exist positive sequences $\left\{R_{m}^{\prime}\right\},\left\{R_{m}^{\prime \prime}\right\},\left\{\tilde{\varepsilon}_{m}\right\}$ satisfying (1.4) such tnat for $R_{m}^{\prime} \leqq t \leqq R_{m}^{\prime \prime}\left(m>m_{0}\right)$, (1.5) is true.

Next, we show that

$$
\lim _{m \rightarrow \infty} \frac{n N\left(r_{m} ; a_{\nu}, f\right)}{\log M\left(r_{m}, f\right)}=\frac{\sin \pi \mu}{\pi \mu}
$$

for some $\nu(\nu=1,2, \cdots, n)$. By the definition of $\chi$,

$$
\varlimsup_{m \rightarrow \infty} \frac{n N\left(r_{m} ; a_{\nu}, f\right)}{\log M\left(r_{m}, f\right)} \leqq \chi .
$$

Thus, if (5.5) is false there exists $\varepsilon(0<\varepsilon<\chi)$ and an unbounded sequence $\Lambda$ of positive integers such that for all $a_{\nu}, \nu=1,2, \cdots, n$,

$$
\frac{n N\left(r_{m} ; a_{\nu}, f\right)}{\log M\left(r_{m}, f\right)}<\chi-\varepsilon \quad(m \in \Lambda) .
$$

Hence applying the reasoning of [8, pp. 230 231] to (3.1) on setting $r=r_{m}$, $R=r_{m}^{\prime \prime} / 2$, we can get

$$
1 \leqq 1-\frac{\varepsilon}{3} \int_{\omega}^{1} x^{\mu} \frac{d x}{(x+1)^{2}}, \quad(m \rightarrow \infty ; m \in \Lambda),
$$

where $\omega=\{(\chi-\varepsilon) /(\chi-\varepsilon / 2)\}^{1 / \mu}$, which is contradiction. Thus, assertion (5.5) is true.

Now we make use of the same process as in [2]. Thus we have, by using (5.1) and (5.5),

$$
\frac{n N\left(r_{m} ; a_{\nu}, f\right)}{\log M\left(r_{m}, f\right)}\left(1+\tilde{\varepsilon}_{m}\right)^{-2} \leqq \frac{n N\left(t ; a_{\nu}, f\right)}{\log M(t, f)} \leqq \frac{n N\left(r_{m} ; a_{\nu}, f\right)}{\log M\left(r_{m}, f\right)}\left(1+\tilde{\varepsilon}_{m}\right)^{2},
$$

provided that $t$ satisfies

$$
R_{m}^{\prime} \leqq t \leqq R_{m}^{\prime \prime} \quad\left(m>m_{0}\right)
$$

Consequently, (5.5) leads to (1.6).

The deduction of (1.7) from (1.6) is a straightforward Tauberian argument.

\section{REFERENCES}

[1] EDREI, A., The deficiencies of meromorphic functions of finite lower order. Duke Math. J., 31 (1946), 1-22.

[2] EDREI, A., Locally tauberian theorems for meromorphic functions of lower order less than one. Trans. Amer. Math. Soc., 140 (1969), 309-332.

[3] Ozawa, M., On the growth of algebroid functions with several deficiencies, II. Kōdai Math. Sem. Rep. 22 (1970), 129-137. 
[4] Nevanlinna, R., Le théorème de Picard-Borel et la théorie des fonctions méromorphes. Paris (1929).

[5] Valiron, G., Sur le minimum du module des fonctions entières d'order inférieur à un. Mathmatica, 11 (1935), 264-269.

[6] Valiron, G., Sur la dérivée des fonctions algebroides. Bull. Soc. Math. 59 (1931), 17-39.

[7] Wahlund, A., Uber einen Zusammenhang zwishen Maximalbetrage der ganzen Funktionen und seiner Grenze nach den Jansenschen Theoreme. Arkıv för Mat. Astr. Fys., 21 A, 23 (1929), 1-34.

[8] Williamson, J., The asymptotic behaviour of a class entire functions of entremal growth. J. London Math. Soc. (2), 7 (1973), 225-234.

Department of Mathematics

Chiba University

YAYOICHO, CHIBA

JAPAN 